\title{
A Novel Method of Isolating Myofibrils From Primary Cardiomyocyte Culture Suitable for Myofibril Mechanical Study
}

Kathleen C. Woulfe ${ }^{1 *}$, Claudia Ferrara ${ }^{2}$, Jose Manuel Pioner ${ }^{2}$, Jennifer H. Mahaffey ${ }^{1}$, Raffaele Coppini ${ }^{2}$, Beatrice Scellini ${ }^{2}$, Cecilia Ferrantini ${ }^{2}$, Nicoletta Piroddi ${ }^{2}$, Chiari Tesi ${ }^{2}$, Corrado Poggesi ${ }^{2}$ and Mark Jeong ${ }^{1}$

${ }^{1}$ Division of Cardiology, Department of Medicine, University of Colorado, Denver, CO, United States, ${ }^{2}$ Division of Physiology, Department of Experimental and Clinical Medicine, University of Florence, Florence, Italy

\section{OPEN ACCESS}

Edited by:

Przemyslaw Blyszczuk,

University of Zurich, Switzerland

Reviewed by:

Akiyoshi Uezumi,

Tokyo Metropolitan Institute of

Gerontology, Japan

Bertrand C.W. Tanner,

Washington State University,

United States

${ }^{*}$ Correspondence:

Kathleen C. Woulfe

Kathleen.woulfe@ucdenver.edu

Specialty section:

This article was submitted to Cardiovascular Biologics and

Regenerative Medicine,

a section of the journal

Frontiers in Cardiovascular Medicine

Received: 30 November 2018 Accepted: 01 February 2019 Published: 19 February 2019

Citation:

Woulfe KC, Ferrara C, Pioner JM, Mahaffey JH, Coppini R, Scellini B,

Ferrantini C, Piroddi N, Tesi $C$,

Poggesi C and Jeong M (2019) A Novel Method of Isolating Myofibrils From Primary Cardiomyocyte Culture

Suitable for Myofibril Mechanical Study. Front. Cardiovasc. Med. 6:12.

doi: 10.3389/fcvm.2019.00012
Myofibril based mechanical studies allow evaluation of sarcomeric protein function. We describe a novel method of obtaining myofibrils from primary cardiomyocyte culture. Adult rat ventricular myocytes (ARVMs) were obtained by enzymatic digestion and maintained in serum free condition. ARVMs were homogenized in relaxing solution (pCa 9.0) with 20\% sucrose, and myofibril suspension was made. Myofibrils were $\mathrm{Ca}^{2+}$-activated and relaxed at $15^{\circ} \mathrm{C}$. Results from ARVM myofibrils were compared to myofibrils obtained from ventricular tissue skinned with Triton X-100. At maximal $\mathrm{Ca}^{2+}$-activation (pCa 4.5) myofibril mechanical parameters from ARVMs were $6.8 \pm 0.9$ $\mathrm{mN} / \mathrm{mm}^{2}$ (resting tension), $146.8 \pm 13.8 \mathrm{mN} / \mathrm{mm}^{2}$ (maximal active tension, $\mathrm{P}_{0}$ ), 5.4 $\pm 0.22 \mathrm{~s}^{-1}$ (rate of force activation), $53.4 \pm 4.4 \mathrm{~ms}$ (linear relaxation duration), 0.69 $\pm 0.36 \mathrm{~s}^{-1}$ (linear relaxation rate), and $10.8 \pm 1.3 \mathrm{~s}^{-1}$ (exponential relaxation rate). Force-pCa curves were constructed from Triton skinned tissue, ARVM culture day 1 , and ARVM culture day 3 myofibrils, and $\mathrm{pCa}_{50}$ were $5.79 \pm 0.01,5.69 \pm 0.01$, and $5.71 \pm 0.01$, respectively. Mechanical parameters from myofibrils isolated from ARVMs treated with phenylephrine were compared to myofibrils isolated from time-matched non-treated ARVMs. Phenylephrine treatment did not change the kinetics of activation or relaxation but decreased the $\mathrm{pCa}_{50}$ to $5.56 \pm 0.03$ (vehicle treated control: 5.67 \pm 0.03 ). For determination of protein expression and post-translational modifications, myofibril slurry was re-suspended and resolved for immunoblotting and protein staining. Troponin I phosphorylation was significantly increased at serine 23/24 in phenylephrine treated group. Myofibrils obtained from ARVMs are a viable method to study myofibril mechanics. Phenylephrine treatment led to significant decrease in $\mathrm{Ca}^{2+}$-sensitivity that is due to increased phosphorylation of $\mathrm{Tnl}$ at serine $23 / 24$. This culture based approach to obtaining myofibrils will allow pharmacological and genetic manipulation of the cardiomyocytes to correlate biochemical and biophysical properties.

Keywords: myofibril, cell culture, mechanics, sarcomere, signaling 


\section{INTRODUCTION}

Since its development, myofibril mechanical assessment has led to advances in understanding of muscle function (1-4). Myofibril-based mechanical assays, compared to studies using larger preparations, offer several advantages. First, as the smallest ensemble of muscle motor proteins, myofibril mechanical experiments provide an evaluation of muscle mechanics without influence by non-motor protein systems such as those involved in calcium handling, energy metabolism, and the extracellular matrix. Second, the level of detail which myofibril mechanical experiments provide, such as resolution of the two phases of cardiac muscle fiber relaxation, is not possible with skinned cardiac fiber or intact cardiomyocyte preparations.

Traditionally, myofibrils are obtained from a small section of muscle that is either glycerinated or skinned in Triton X-100 and homogenized $(5,6)$. A major limitation of this approach is that these myofibrils are "as-is" with limited potential for biochemical manipulations. In this regard, specifically targeting a particular signaling pathway can only be achieved by generating transgenic animals, which is time-intensive and costly. The major strength of cardiomyocyte culture-based experiments is the ability to utilize readily available reagents and tools to address the myofilament mechanical consequences of altering a particular cellular function. Furthermore, with rapid advancement in stem cell-derived cellular systems, an in vitro method of obtaining myofibrils will provide a powerful experimental platform to better understand the pathobiology of diseases involving striated muscle. In this paper, we report a novel method of obtaining myofibrils from primary adult rat ventricular myocyte (ARVM) culture. We show that myofibrils obtained from primary ARVMs are equivalent to the traditional method and show applicability of this method to dissect the functional consequences of manipulating a specific signaling cascade.

\section{METHODS}

\section{Experimental Protocol}

Adult rat left ventricular myocytes (ARVMs) were obtained from female Sprague Dawley rats (250-300 g) (7). Animal studies were reviewed and approved by University of Florence and University of Colorado Institutional Animal Care and Use Committee (IACUC) thereby meeting the standards set by the Directive 2010/63/EU of the European Parliament on the protection of animals used for scientific purposes and the NIH standards for the care and use of laboratory animals. The heart was rapidly removed and retrograde perfused with perfusion buffer $(120.5 \mathrm{mM} \mathrm{NaCl}, 14.7 \mathrm{mM}$ $\mathrm{KCl}, 0.6 \mathrm{mM} \mathrm{KH} \mathrm{PO}_{4}, 0.6 \mathrm{mM} \mathrm{Na}_{2} \mathrm{HPO}_{4}, 1.2 \mathrm{mM} \mathrm{MgSO}_{4}$, $4.6 \mathrm{mM} \mathrm{NaHCO} 3,10 \mathrm{mM}$ Na-HEPES, $30 \mathrm{mM}$ Taurine, $10 \mathrm{mM}$ 2,3-butanedione monoxime, $5.5 \mathrm{mM}$ Glucose, $\mathrm{pH}$ 7.2) for $10 \mathrm{~min}$ at $37^{\circ} \mathrm{C}$. A small section of the left ventricular apex was cut at the end of the pre-digestion perfusion. The small apical tissue was skinned in Triton X-100. The remainder of the heart was enzymatically digested to make ARVMs (Figure 1).

\section{Cardiomyocyte Culture}

The heart was digested with Liberase DH (Roche, $0.33 \mathrm{mg} / \mathrm{ml}$ ) for $8 \mathrm{~min}$, cut into small pieces, and a slurry was filtered through sterile $150-\mathrm{nm}$ mesh. The filtrate was centrifuged at $400 \mathrm{~g}$ for $4 \mathrm{~min}$ to separate myocytes from non-myocytes. The myocyte suspension was layered over $60 \mu \mathrm{g} / \mathrm{ml}$ of BSA and allowed to settle for $15 \mathrm{~min}$ to separate myocytes from non-myocytes. Myocyte concentration was determined and plated on $100 \mathrm{~mm}$ laminin-coated plastic culture dishes at a density of 100 to 150 cells $/ \mathrm{mm}^{2}$. The ARVM culture was maintained in serumfree DMEM supplemented with albumin $(2 \mathrm{mg} / \mathrm{ml})$, carnitine ( $2 \mathrm{mmol} / \mathrm{l})$, creatine $(5 \mathrm{mmol} / \mathrm{l})$, taurine $(5 \mathrm{mmol} / \mathrm{l}), \mathrm{BDM}(1$ $\mathrm{mg} / \mathrm{ml})$, and penicillin-streptomycin $(100 \mu \mathrm{g} / \mathrm{ml})$.

\section{Myofibrils From Cardiomyocyte Culture}

ARVMs were washed in sterile PBS (room temperature) twice then collected one of two ways. (1) The cells were collected in $1 \mathrm{ml}$ of cold $20 \%$ sucrose in relaxing solution (pCa 9.0) with protease inhibitor cocktail $(10 \mu \mathrm{M}$ leupeptin, $5 \mu \mathrm{M}$ pepstatin, $200 \mu \mathrm{M}$ phenyl-methylsuphonylfluoride, $10 \mu \mathrm{M}$ E64, $500 \mu \mathrm{M}$ NaN3, $2 \mathrm{mM}$ dithioerythritol). The demembraned ARVM slurry was vortexed $(30 \mathrm{~s}$ then settled on ice for $10 \mathrm{~min}$. The cell suspension was centrifuged ( $300 \mathrm{~g}$ for $15 \mathrm{~min}$ in $4^{\circ} \mathrm{C}$ ) and the pellet was resuspended in fresh relaxing solution with protease inhibitor cocktail to remove the sucrose. The resuspension/wash cycle was repeated twice. The final demembraned ARVMs were resuspended in relaxing solution with protease inhibitor cocktail and homogenized at medium speed (Tissue Tearer) for 15-20 s to make the final myofibril suspension. (2). Cells were collected in $1 \mathrm{ml}$ of cold $0.01 \%$ Triton-X in relaxing solution (pCa9.0) with protease inhibitor cocktail. The ARVM slurry was treated as described above.

\section{Myofibrils From Apical Tissue}

The left ventricular apex was cut into thin slices and bathed in $0.05 \%$ Triton X-100 and protease inhibitor cocktail in rigor solution overnight at $4{ }^{\circ} \mathrm{C}$. The skinned tissue was then washed twice in rigor solution with protease inhibitor cocktail with gentle shaking. The skinned tissue was resuspended in $1 \mathrm{ml}$ rigor solution with protease inhibitor cocktail and homogenized at medium speed for $15-20 \mathrm{~s}$ to make the final myofibril suspension $(8,9)$.

\section{Myofibril Mechanical Study}

We used previously published techniques to measure and control the force and length of isolated myofibrils activated and relaxed by fast solution switching $(8,9)$. Briefly, a small volume of myofibril suspension was transferred to a temperature controlled chamber $\left(15^{\circ} \mathrm{C}\right)$ filled with relaxing solution (pCa 9.0). A small bundle of myofibrils were mounted between two microtools. One tool was connected to a motor that could produce rapid length changes (Mad City Labs). The second tool was a calibrated cantilevered force probe $(4-8 \mu \mathrm{m} / \mu \mathrm{N}$; frequency response $2-$ $5 \mathrm{KHz}$ ). Myofibrils were set 5-10\% above slack myofibril length in relaxed conditions ( $\mathrm{pCa} 9$ ). Average sarcomere length and myofibril diameter were measured using ImageJ. Mounted myofibrils were activated and relaxed by rapidly translating the 


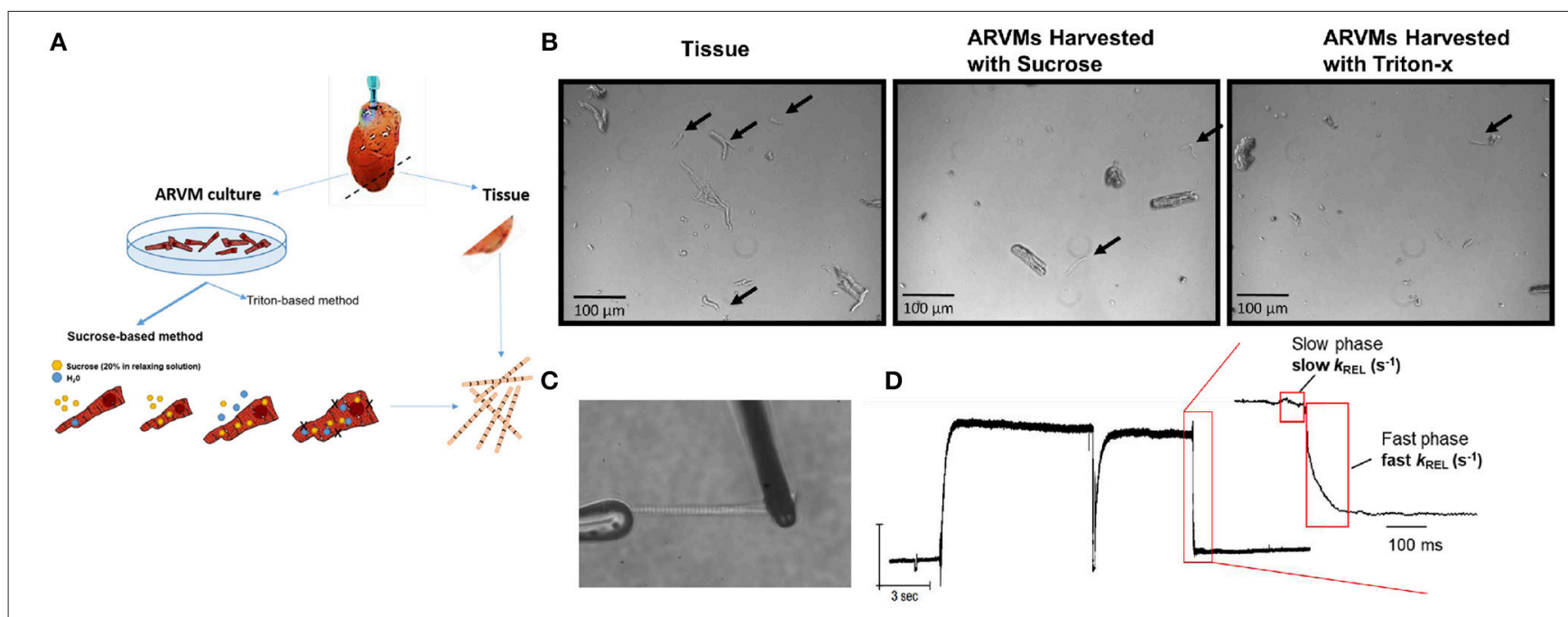

FIGURE 1 | (A) Experimental scheme: hearts from Sprague Dawley rats are retrograde perfused. Before enzymatic digestion, a small section of the apex is cut to obtain myofibrils by skinning in Triton-X100. Remaining heart was digested in Liberase DH to obtained primary ARVM culture. Myofibrils from ARVM culture were obtained by sucrose based osmotic shock method. (B) Representative images of 10x magnification demonstrating the different quantity and quality of myofibrils isolated using the different techniques. Black arrows indicate useable myofibrils in each field. The myocyte fragments shown in the panel of ARVMs harvested using Triton-x show a morphology distinct from the fragments of the other harvest techniques. (C) Representative image of a myofibril isolated from ARVMs using the sucrose-based method. (D) Representative trace from an ARMV-derived myofibril activated and relaxed by fast solution switching. ARVM, adult rat ventricular myocyte.

interface between two flowing streams of solutions of different pCa $(9,10)$. Data was collected and analyzed using a customized LabView software. Measured mechanical and kinetic parameters were defined as follows: resting tension $\left(\mathrm{mN} / \mathrm{mm}^{2}\right)-$ myofibril basal tension in fully relaxing condition ( $\mathrm{pCa} 9.0$ ); maximal tension $\left(\mathrm{mN} / \mathrm{mm}^{2}\right)$-maximal tension generated at full calcium activation ( $\mathrm{pCa} 4.5$ ); the rate constant of tension development following maximal calcium activation $\left(k_{\mathrm{ACT}}\right)$; the rate constant of tension redevelopment following a release-restretch applied to the activated myofibril $\left(k_{\mathrm{TR}}\right)(11)$; rate constant of early slow force decline (slow $k_{\mathrm{REL}}$ )-the slope of the linear regression normalized to the amplitude of relaxation transient, duration of early slow force decline-measured from onset of solution change to the beginning of the exponential force decay, the rate constant of the final exponential phase of force decline (fast $k_{\mathrm{REL}}$ ). To determine force-pCa relationship, peak isometric force was recorded at maximal ( $\mathrm{pCa} 4.5$ ) and submaximal ( $\mathrm{pCa} 5.2,5.4$, $5.6,5.8,7)$ calcium concentrations and fitted to the Hill equation $\left(\mathrm{P} / \mathrm{P} 0=1 /\left(1+10^{(-\mathrm{nh}(\mathrm{pCa} 50-\mathrm{pCa}))}\right)\right.$.

\section{Myofilament Protein Analysis}

To determine the level of phosphorylated myofilament proteins, the myofibril slurry was spun down and protein pellet was resuspended in isoelectric focusing buffer (IEF; $8 \mathrm{M}$ Urea, $2.5 \mathrm{M}$ Thiourea, 4\% Chaps, $2 \mathrm{mM}$ EDTA, $1 \mathrm{mM}$ DTT, $1 \%$ TBP, phosphatase, and protease inhibitors). Twenty micrograms of protein was resolved by SDS-PAGE using a $10 \%$ gel and stained with ProQ Diamond. The gel was washed in methanol and restained with commassie brilliant blue (CBB) to determine total protein abundance. Gels were scanned and densitometry was measured using ImageJ. To determine the site-specific phosphorylation of troponin I, Western blots were performed. Site specific phospho-antibodies directed to serine 23/24 (Phosphosolutions p2010-2324, 1:1,000) and serine 43/44 (Phosphosolutions 9056, 1:1,000) of troponin I were used for Western blotting. TnI antibody (Fitzgerald 10R-T123K, 1:1,000) was used to determine the relative abundance of phosphorylated proteins. Densitometry data was used to normalize the phosphospecific signal over the total TnI signal.

\section{Statistical Analysis}

Statistical analyses were performed using Prism Version 6 (GraphPad Software, San Diego, CA). Mean \pm SEM values are presented. Data were checked for normality by Shapiro-Wilks test and if normal, compared by Student $t$-test ( 2 unpaired groups) or one-way ANOVA (>2 groups) with Newman-Keuls post-test. If not normal, data were log-transformed before statistical testing. Probability values of $p<0.05$ were considered significant.

\section{RESULTS}

\section{Myofibrils From Cultured Cardiomyocytes Permeabilized Using Sucrose Are Viable}

Our initial aim was to determine the cardiomyocyte skinning buffer and homogenization protocol to harvest myofibrils from ARVMs. We began with low concentrations of Triton X-100 in rigor or relaxing solutions. $0.1 \%$ Triton X-100 caused complete lysis of cardiomyocytes in either buffer. We were able to harvest myofibrils from cardiomyocytes skinned in $0.01 \%$ Triton X100 in rigor solutions but yielded poor quality and lower quantity of myofibrils. These myofibrils tended to be shorter, more fragmented, and difficult to mount on our force recording 
machine (Figure 1B). Sucrose (20\% by volume) in relaxing solution was able to disrupt the cellular membrane without affecting the integrity of the myofibrils (Figures 1B-D).

\section{Mechanical Parameters From Myofibrils From ARVM Are Comparable to the Mechanical Parameters From Myofibrils From Tissue}

Next mechanical parameters of myofibrils from ARVM culture harvested with sucrose or frozen tissue were compared. Myofibrils were isolated from frozen rat LV as a comparison since this is the standard source of myofibrils used to assess disease-specific differences in human hearts or animal models. The isometric sarcomere length measured was similar. The

TABLE 1 | Comparison of mechanical parameters between myofibrils obtained from ARVM culture vs. traditional small tissue section.

\begin{tabular}{lccc}
\hline & ARVM $(\mathbf{N}, \boldsymbol{n})$ & Tissue $(\mathbf{N}, \boldsymbol{n})$ & $\boldsymbol{P}$ \\
\hline Sarcomere length $(\mu \mathrm{m})$ & $2.24 \pm 0.09(6,19)$ & $2.28 \pm 0.07(6,15)$ & $\mathrm{ns}$ \\
Resting tension $\left(\mathrm{mN} / \mathrm{mm}^{2}\right)$ & $6.8 \pm 3.5(6,17)$ & $6.6 \pm 3.8(6,13)$ & $\mathrm{ns}$ \\
Maximal tension $\left(\mathrm{mN} / \mathrm{mm}^{2}\right)$ & $146.8 \pm 51.7(6,14)$ & $139.6 \pm 51.3(6,15)$ & $\mathrm{ns}$ \\
$K_{\text {ACT }}\left(\mathrm{s}^{-1}\right)$ & $5.37 \pm 0.95(6,18)$ & $4.97 \pm 1.14(6,14)$ & $\mathrm{ns}$ \\
$K_{\text {TR }}\left(\mathrm{s}^{-1}\right)$ & $5.20 \pm 0.89(6,17)$ & $4.52 \pm 0.82(6,15)$ & $p<0.05$ \\
Linear relaxation duration $(\mathrm{ms})$ & $53.4 \pm 17.7(6,16)$ & $52.0 \pm 16.2(6,12)$ & $\mathrm{ns}$ \\
Exponential relaxation rate $\left(\mathrm{s}^{-1}\right)$ & $10.8 \pm 4.8(6,14)$ & $14.6 \pm 6.5(6,12)$ & $\mathrm{ns}$ \\
\hline
\end{tabular}

Myofibrils are fully calcium activated ( $\mathrm{pCa} 4.5$ ) and fully relaxed ( $\mathrm{pCa} 9$ ) by fast solution switching. Data presented with mean \pm SEM. ( $N, n)$, (N\# of rats, $n$ \# of myofibrils). mechanical and kinetic behavior of ARVM myofibrils was not statistically different from tissue-derived myofibrils for most parameters (Table 1). The kinetics of force redevelopment $\left(k_{\mathrm{TR}}\right)$ were, however, slower in myofibrils from tissue. Mechanical rundown, the decrement of force generation, and its kinetics with successive activation-relaxation cycles was comparable between myofibrils isolated from ARVMs and tissue (data not shown). Force-pCa curves were constructed from myofibrils obtained from ARVMs in culture or from tissue. $\mathrm{Ca}^{2+}$-sensitivity and the Hill coefficients $(\mathrm{nH})$ were similar between myofibrils from ARVM and tissue (Figure 2A).

\section{Duration of Cultured Cells Affects Kinetics of Activation and Relaxation but Not Calcium Sensitivity}

In order to understand how prolonged culture affects the mechanical properties of cardiac myofibrils, we compared myofibrils isolated using sucrose from cardiomyocyte culture at day 1 and day 3. Myofibrils from day 1 group were isolated from cardiomyocytes that were kept in serum-free media in a $37^{\circ} \mathrm{C}$ incubator between 2 and $8 \mathrm{~h}$. The 3 -day-old cardiomyocytes were kept in a serum-free culture environment for an additional $48 \mathrm{~h}$. Although magnitude of tension generation was unaffected by long-term culture, 3 days of culture showed changes in kinetics of activation and relaxation (Table 2). Using Pro-Q Diamond staining to assess for phosphorylation of sarcomeric proteins, we did not see a significant change in the degree of phosphorylation of key contractile and regulatory proteins of the
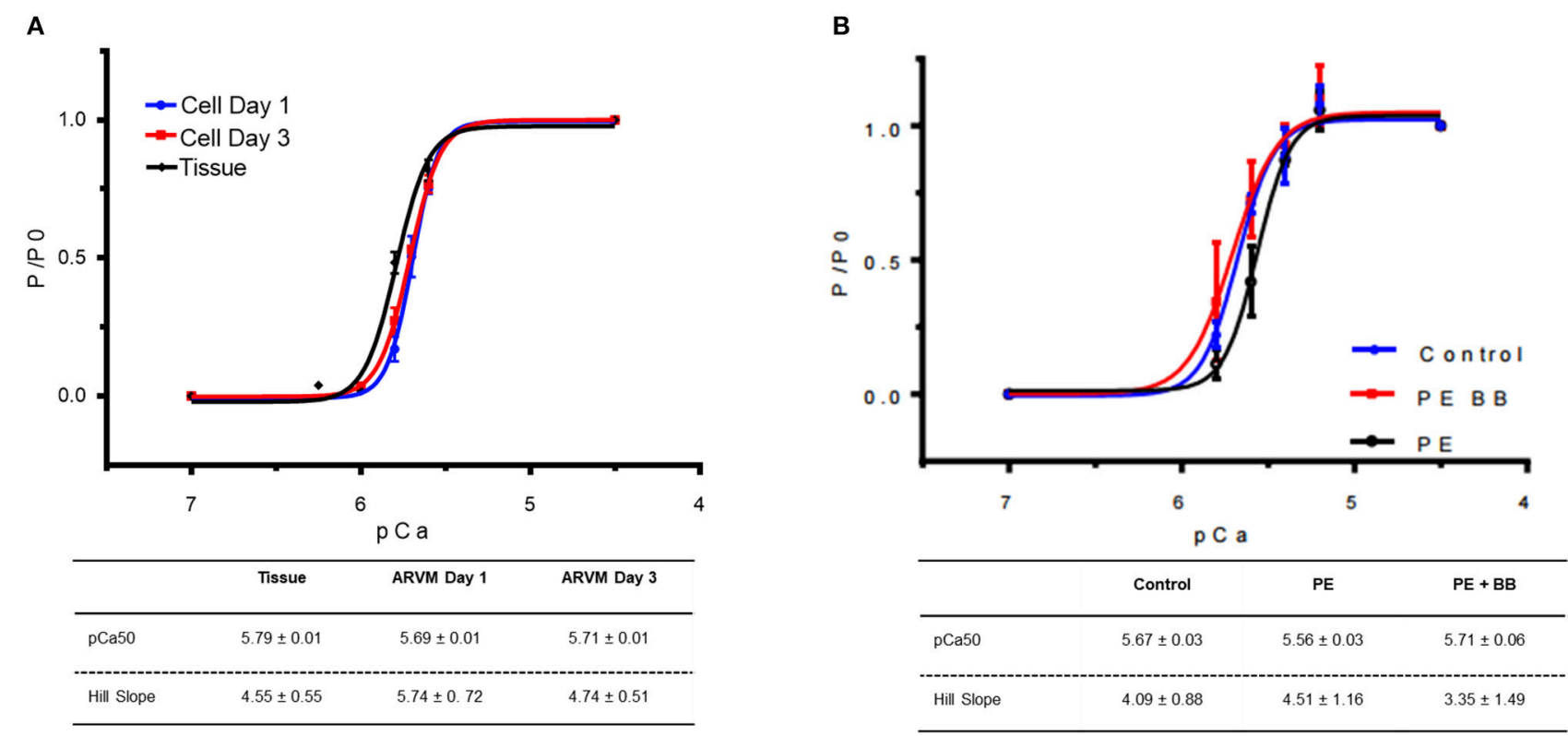

FIGURE 2 | (A) Force-pCa relationship of myofibrils obtained from cardiomyocyte culture day 1 (blue), culture day 3 (red) vs. myofibrils isolated from cardiac tissue (black). At each pCa, maximal tension was normalized to tension generated at pCa $4.5\left(P_{0}\right)$. Data points are mean \pm SEM. Lines are drawn according to the parameters estimated by fitting to the Hill equation $\left(P / P_{0}=1 /\right)\left(1+10^{(-n h(p C a 50-p C a))}\right)$. N = 4 rats, $16-20$ myofibrils. (B) Force-pCa relationship of myofibrils obtained from ARVMs that were PE-treated (black), myofibrils treated with PE and the $\beta$-blocker propranolol (BB) (red) vs. vehicle treated control (blue). $N=6$ rats, 16-22 myofibrils. ARVM, adult rat ventricular myocyte; PE, phenylephrine; BB, $\beta$-blocker propranolol. 
TABLE 2 | Comparison of mechanical parameters between myofibrils obtained from ARVMs cultured for 1 day and ARVMs maintained in serum-free medium for 3 days.

\begin{tabular}{lccc}
\hline & ARVM Day 1 (N, $\boldsymbol{n})$ & ARVM Day 3 (N, $\boldsymbol{n})$ & $\boldsymbol{P}$ \\
\hline Sarcomere length $(\mu \mathrm{m})$ & $2.24 \pm 0.09(6,19)$ & $2.25 \pm 0.07(8,9)$ & $\mathrm{ns}$ \\
Resting tension $\left(\mathrm{mN} / \mathrm{mm}^{2}\right)$ & $6.8 \pm 3.5(6,17)$ & $7.3 \pm 3.2(8,19)$ & $\mathrm{ns}$ \\
Maximal tension $\left(\mathrm{mN} / \mathrm{mm}^{2}\right)$ & $146.8 \pm 51.7(6,14)$ & $135.8 \pm 47.6(8,18)$ & $\mathrm{ns}$ \\
$K_{\text {ACT }}\left(\mathrm{s}^{-1}\right)$ & $5.37 \pm 0.95(6,18)$ & $4.05 \pm 0.98(8,19)$ & $P<0.05$ \\
$K_{\text {TR }}\left(\mathrm{s}^{-1}\right)$ & $5.20 \pm 0.89(6,17)$ & $4.90 \pm 0.95(8,19)$ & $\mathrm{ns}$ \\
Linear relaxation duration $(\mathrm{ms})$ & $53.4 \pm 17.7(6,16)$ & $34.2 \pm 8.5(8,14)$ & $P<0.05$ \\
Exponential relaxation rate $\left(\mathrm{s}^{-1}\right)$ & $10.8 \pm 4.8(6,14)$ & $10.2 \pm 5.2(8,16)$ & $\mathrm{ns}$ \\
\hline
\end{tabular}

Myofibrils are fully calcium activated ( $\mathrm{pCa} 4.5)$ and fully relaxed ( $\mathrm{pCa} 9$ ) by fast solution switching. Data presented with mean \pm SEM.

sarcomere (Figure 3). The duration that ARVMs were kept in primary culture had no impact on $\mathrm{Ca}^{2+}$-sensitivity or the Hill coefficient (Figure 2A).

\section{Treating ARVMs With Phenylephrine Leads to Decreased Calcium Sensitivity in Myofibrils Due to Phosphorylation of Troponin I}

To demonstrate the applicability of culture based cardiomyocyte myofibrils to study the effect of manipulating the adrenergic signaling pathway, we treated ARVMs with phenylephrine $(\mathrm{PE} ; 20 \mu \mathrm{M})$ for $48 \mathrm{~h}$ and performed myofibril mechanical measurements and sarcomeric protein analysis. Myofibrils were harvested using the sucrose method. We and others have shown that prolonged PE treatment leads to significant cardiomyocyte hypertrophy, induction of the pathologic gene profile, and deterioration of intact cardiomyocyte function $(12,13)$. The kinetics of activation and relaxation, and tension generation were unchanged with PE treatment (Table 3). Treatment with PE led to a significant decrease in calcium sensitivity (Figure 2B). Using CBB protein staining, we did not see a change in the expression level of sarcomeric proteins between vehicle vs. PE treated ARVMs (Figure 3). Global phosphorylation status was examined using Pro-Q Diamond stain. PE treatment led to increased phosphorylation of troponin I (TnI) (Figure 3). Western blot analysis confirmed the change in phosphorylation of serine 23/24 while serine 44 was not affected (Figure 4A). Decreased calcium sensitivity due to phosphorylation of TnI at serine $23 / 24$ is consistent with previously published studies. To confirm our hypothesis that PE was also activating PKA via $\beta$-adrenergic signaling pathway, we treated ARVMs with $P E$ with or without propranolol $(2 \mu \mathrm{M})$. Propranolol treatment was sufficient to inhibit the increased phosphorylation of Serine 23/24 TnI (Figure 4) and the shift in pCa50 (Figure 2B). Propranolol treatment alone did not have any effect in basal expression of TnI or phosphorylation (Figure 4).

\section{DISCUSSION}

We report a novel method of obtaining myofibrils from cultured ARVMs which are suitable for myofibril mechanical experiments.
We establish that myofibrils obtained from an ARVM culture system are equivalent in mechanical properties to myofibrils prepared by skinning small cardiac tissue with Triton-X100. Demonstrating applicability of this method, we show that PE treatment does not significantly affect activation kinetics and tension generation, but PE treatment increases phosphorylation of TnI contributing to decreased myofilament calcium sensitivity when compared to vehicle treated controls. These findings show that primary ARVM culture system can be used for myofibril mechanical studies, and will expand the utility of myofibril mechanical experiment by providing a wider palette of experimental conditions.

The major advantage of myofibrils obtained from a primary culture system over the traditional Triton X-100 method is that primary culture system allows manipulation of specific cellular processes using widely available experimental techniques. The traditional method of obtaining myofibrils from a small heart section can be viewed as "as-is" with limited potential for exvivo manipulation. The combination of myofibril mechanical experiments and the wide array of biochemical techniques available to manipulate cultured cells provides a flexible experimental system to study the mechanical consequences of altering a specific cellular function. ARVM based myofibril studies provides additional advantages. ARVM are relatively inexpensive when compared to the need for generating mutant animals. For example, if you consider a potential experiment studying how protein $\mathrm{x}$ impacts myofibril mechanics, the cost and time to generate a transgenic or knockout animal is roughly $\$ 7,000$ and can take up to 14 weeks. From there, there are costs associated with breeding and maintenance of a colony where animal costs can exceed $\$ 250$ a month not including the salary of the staff necessary to oversee the colony. This total quickly reaches $\$ 10,000$ or more and takes at least 6 months. In contrast, consider purchasing 6 adult rats at roughly $\$ 50$ each and generating ARVMs. From here, the cells can be treated with inhibitors or ectopically expressed proteins and myofibril mechanics studied. On a practical level, we found that homogenizing intact cardiomyocytes to small bundles of myofibrils was easier to achieve from ARVMs than from small cardiac tissue. Our ARVM based method is complementary to the current standard and provides an additional source of experimental material. Importantly, our results show that myofibrils from ARVMs are mechanically comparable to myofibrils derived from tissue.

One difference in mechanical properties that we noted between tissue and ARVMs harvested using sucrose was that $\mathrm{k}_{\mathrm{TR}}$ was faster in ARVMs. This difference could be due to structural changes in the sarcomeres that occur following plating $(14,15)$ or it may be an experimental difference in isolation techniques (Triton $\mathrm{X}-100$ compared to sucrose). It is important to recognize that these factors affect the mechanical parameters and experimental conditions should be considered appropriately. To further explore how plating impacts sarcomere function, we determined how duration of primary culture impacts myofibril function. Cardiomyocytes have been found to undergo transformation when maintained in primary culture. Although maintaining cardiomyocytes for up to 15 days has 


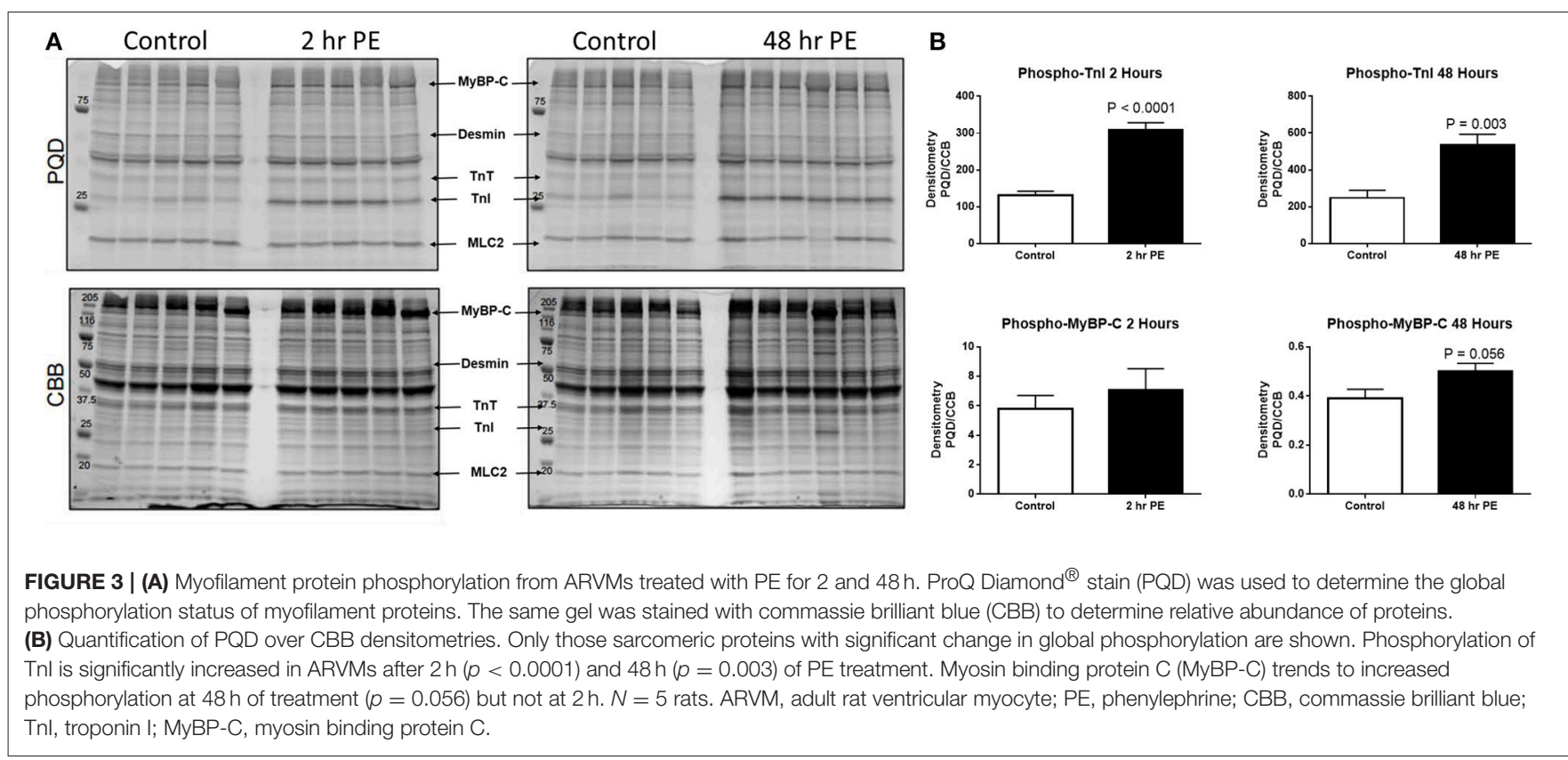

TABLE 3 | Comparison of mechanical parameters between myofibrils from ARVMs treated with PE for $48 \mathrm{~h}$.

\begin{tabular}{lccc}
\hline & Vehicle $(\mathbf{N}, \boldsymbol{n})$ & PE (N, $\boldsymbol{n})$ & $\boldsymbol{P}$ \\
\hline $\begin{array}{l}\text { Sarcomere length } \\
(\mu \mathrm{m})\end{array}$ & $2.21 \pm 0.1(6,19)$ & $2.21 \pm 0.1(6,22)$ & $\mathrm{ns}$ \\
$\begin{array}{l}\text { Resting tension } \\
\left(\mathrm{mN} / \mathrm{mm}^{2}\right)\end{array}$ & $7.6 \pm 2.8(6,18)$ & $6.7 \pm 2.5(6,17)$ & $\mathrm{ns}$ \\
$\begin{array}{l}\text { Maximal Tension } \\
\left(\mathrm{mN} / \mathrm{mm}^{2}\right)\end{array}$ & $135.8 \pm 47.6(6,17)$ & $118.8 \pm 38.6(6,17)$ & $\mathrm{ns}$ \\
$K_{\text {ACT }}\left(\mathrm{s}^{-1}\right)$ & $4.05 \pm 0.98(6,19)$ & $3.95 \pm 0.94(6,19)$ & $\mathrm{ns}$ \\
$K_{\text {TR }}\left(\mathrm{s}^{-1}\right)$ & $4.90 \pm 0.95(6,19)$ & $4.37 \pm 1.02(6,19)$ & $P=0.059$ \\
Linear Relaxation & $34.2 \pm 8.5(6,15)$ & $43.8 \pm 17.1(6,15)$ & $\mathrm{ns}$ \\
$\begin{array}{l}\text { Duration }(\mathrm{ms}) \\
\text { Linear Relaxation Rate }\left(\mathrm{s}^{-1}\right)\end{array}$ & $1.27 \pm 0.91(6,6)$ & $1.35 \pm 0.65(6,8)$ & $\mathrm{ns}$ \\
$\begin{array}{l}\text { Exponential Relaxation } \\
\text { Rate }\left(\mathrm{s}^{-1}\right)\end{array}$ & $9.04 \pm 3.3(6,15)$ & $10.0 \pm 5.1(6,19)$ & $\mathrm{ns}$ \\
& & &
\end{tabular}

Myofibrils are fully calcium activated ( $\mathrm{pCa} 4.5)$ and fully relaxed ( $\mathrm{pCa} 9$ ) by fast solution switching. Data presented with mean \pm SEM. ( $N, n)$, ( $N \#$ of rats, $n$ \# of myofibrils).

been described (15), we and other investigators report an optimal culture time frame of up to 5-8 days (7). It is believed that the limited life-span of ARVMs in culture is due to dedifferentiation. Eppenberger et al. (16) demonstrated that over time cultured ARVMs have extensive morphological changes so that they look more like fetal myofibril and therefore achieve a less differentiated state (16). Dedifferentiation of cardiomyocytes in long-term culture is inevitable, but serum-free culture media decreases the rate of dedifferentiation (14). The culture media is critical to maintain healthy cells, and the culture media additives for ARVMs are albumin, creatine, carnitine, taurine, BDM, penicillin, and streptomycin. We have set 5 days as the upper limit for maintaining ARVMs in culture, and have designed our experiments around this time frame. We and others have previously shown and show additional data in this report that key functional and regulatory proteins of the sarcomere are unchanged in the level of expression and modification $(12,13$, 17). The half-life of sarcomeric proteins ranges between 3 and 10 days $(18,19)$. Our data shows that there is no significant change in the expression level or global phosphorylation of sarcomeric proteins obtained from ARVMs in short vs. long term culture. Although we do not report qualitative or quantitative changes of sarcomeric proteins, we report significant changes in activation and relaxation kinetics between day 1 and day 3 . This degree of change in biomechanical properties is not unexpected considering that ARVMs in primary culture are dedifferentiating. We hypothesize that routine biochemical assays used in this study to evaluate for the abundance and the post-translational modification status of sarcomeric proteins are not sensitive enough to detect subtle changes. These small changes appear to be sufficient to exert significant change in myofilament function. In this regard, experiments that compare myofibril mechanics between freshly isolated ARVM to one that is several days old would be inappropriate. In our experimental protocol, treated groups are always compared to age-matched vehicle treated controls, thereby allowing us to evaluate the effects of the treatment group while taking into account the unavoidable changes that are inherent in a primary culture based experiment.

Adult rat ventricular cardiomyocytes treated with PE $(20 \mu \mathrm{M})$ for $48 \mathrm{~h}$ demonstrate that TnI phosphorylation is an important effector of sarcomeric protein function. Previously, we and others have shown that treating ARVMs with $48 \mathrm{~h}$ of PE caused contractile dysfunction (7, 13). This report builds on the previous study and provides new data showing that cardiomyocyte dysfunction does not require dysfunction of myofibril contractile machinery. This observation suggests a hierarchy of cardiomyocyte dysfunction by showing that the contractile machinery remains capable even in the face of 


\section{A}

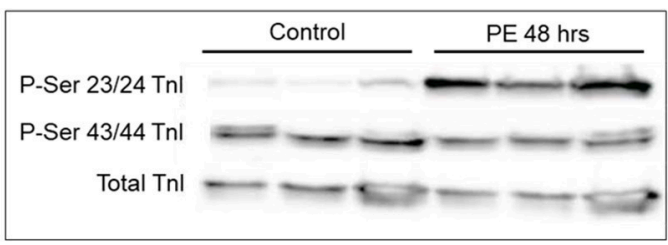

B

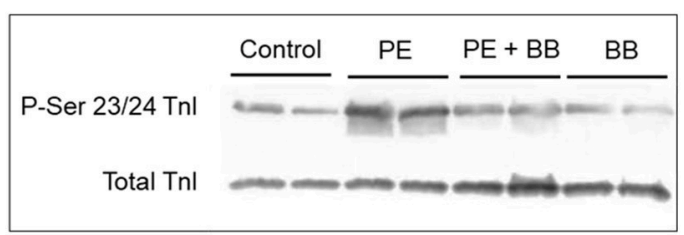

C

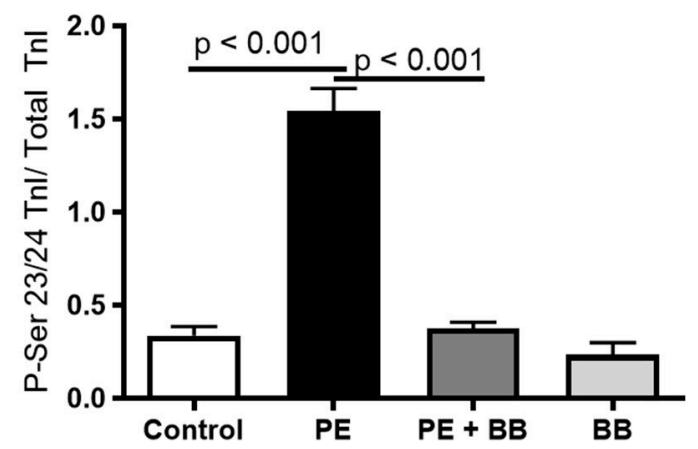

FIGURE 4 | (A) Representative Western blot demonstrating serine 23/24 is preferentially phosphorylated with PE treatment. Each lane is protein isolated from different experiments. These conditions were repeated in ARVMs cultured from 5 different rat hearts. (B) Representative image of a Western blot showing pre-treatment of ARVMs with the $\beta$-blocker propranolol (BB) leadsto inhibition of $\mathrm{Tnl}$ phosphorylation at serine 23/24. Experiment was repeated in ARVMs cultured from 3 different rat hearts. (C) Quantification of Tnl serine 23/24 phosphorylation normalized to total Tnl. PE significantly increases phosphorylation of Tnl at Ser 23/24 $(p<0.001)$. Treatment with PE and a $\beta$-blocker (BB) propranolol, normalizes Tnl phosphorylation (PE tx $\vee$ PE+BB $p<0.001)$. Each lane is protein isolated from different experiments. These conditions were repeated in ARVMs cultured from 3 different rat hearts. Tnl, troponin I; PE, phenylephrine; ARVM, adult rat ventricular myocyte; BB, $\beta$-blocker propranolol.

cellular dysfunction. If the basic contractile unit from a dysfunctional cardiomyocyte is functioning normally, then can we target the basic contractile unit to normalize the whole cell function? We have previously published data showing that targeted inhibition of histone deacetylase 6 can augment contractility of myofibrils to normalize whole heart function in an angiotensin model of rodent heart failure (20). This strategy of targeting the myofibril to augment inotrope may offer benefits over currently available agents such as dobutamine or milrinone which targets the upstream receptor or enzymatic cascade immediately below the receptor. Clinical data may support this hypothesis since in adult heart failure literature, prolonged use of dobutamine or milrinone, while having positive inotropic effects, leads to increased mortality from side effects $(21,22)$. If our observation can be applied to human heart failure, perhaps targeting the myofibrillar proteins directly may provide a more direct and less toxic inotropic support.

We also report that $\mathrm{PE}$, an $\alpha$-adrengeric and $\beta$-adrenergic agonist (23), modifies $\mathrm{TnI}$ at serine 23/24. TnI serine 23/24 is predominantly under the control of protein kinase A (PKA), the down-stream effector of $\beta$-adrenergic receptor cascade. This observation provides insight into the role of PKA in modulating troponin I phosphorylation, the kinetics of cross-bridge cycle, and calcium sensitivity of cardiac myofibrils. We show that increased phosphorylation of serine 23/24 TnI decreases the calcium sensitivity without affecting the kinetics of activation or relaxation. Our results are in agreement with previous work performed in human myofibrils treated with PKA (24). Others, using rodent myofibrils, showed that activation of PKA resulted in both right shift in calcium sensitivity and changes in activation and relaxation kinetics $(25,26)$. Specifically, Rao et al. (25) exchanged TnI with targeted mutations in serine 23/24 into myofibrils to illustrate that phosphorylation status of TnI can control both relaxation duration and calcium sensitivity of activation. While both studies show the regulatory role of serine 23/24 in calcium sensitivity of activation, PE treatment of ARVMs leads to different results in relaxation kinetics. This difference may be due to the experimental condition. In our experiment, we stimulated cardiomyocytes with an upstream agonist thereby activating myriad of other secondary effectors. In Rao's work, exchanging mutant TnI into myofibrils would avoid the effects of signaling cascade that results from receptor activation (25). Regardless of the experimental protocol, we are in agreement that serine $23 / 24$ is an important effector in changing sensitivity to calcium activation. Additional conclusions about the role of serine 23/24 in regulating relaxation remains, however, unsettled. The ability to obtain viable myofibrils from ARVMs presents an opportunity to use established myofibril mechanical assays in an in vitro model system which will be a valuable tool to inform mechanisms driving mechanical alterations.

In conclusion, we report that myofibrils obtained from ARVM culture are similar in mechanical properties to myofibrils isolated by skinningsmall sections of heart tissue.. While the traditional method of Triton X-100 based myofibrils will remain germane to myofibril mechanical research, we are confident that the primary ARVM culture based myofibrils will prove to be a powerful research tool complementing the time proven traditional method.

\section{AUTHOR CONTRIBUTIONS}

MJ, CFa, JM, BS, KW, and NP performed myofibril mechanical experiments. MJ, JP, RC, and CFi isolated adult rat ventricular myocytes and myofibrils. MJ and CP designed and built the photodiode motion detector. $\mathrm{MJ}, \mathrm{CFi}$, and $\mathrm{RC}$ wrote the data acquisition program. MJ and JM performed protein analysis. MJ, $\mathrm{KW}, \mathrm{CT}$, and CP designed the experiments and analyzed all data. All authors contributed to writing the manuscript. 


\section{FUNDING}

This work was supported by Sarnoff Endowment [Fellow to Faculty Transition 379 Award (MJ)]; the National Institute of Health [2K12HD057022-11 (KW); 380 5KL2TR001080-02 (MJ)]; EU [STREP project 241577 BIG HEART, seventh 381 European Framework Program (CP)]; and the Italian Ministry of Health [WFR GR-2011-382 02350583 (CFi)].

\section{REFERENCES}

1. Tesi C, Piroddi N, Colomo F, Poggesi C. Relaxation kinetics following sudden $\mathrm{Ca}(2+)$ reduction in single myofibrils from skeletal muscle. Biophys J. (2002) 83:2142-51. doi: 10.1016/S0006-3495(02)73974-X

2. Stehle R, Kruger M, Pfitzer G. Force kinetics and individual sarcomere dynamics in cardiac myofibrils after rapid ca(2+) changes. Biophys J. (2002) 83:2152-61. doi: 10.1016/S0006-3495(02)73975-1

3. Stehle R, Solzin J, Iorga B, Poggesi C. Insights into the kinetics of Ca2+regulated contraction and relaxation from myofibril studies. Pflugers Arch. (2009) 458:337-57. doi: 10.1007/s00424-008-0630-2

4. Stehle R, Iorga B. Kinetics of cardiac sarcomeric processes and rate-limiting steps in contraction and relaxation. J Mol Cell Cardiol. (2010) 48:843-50. doi: 10.1016/j.yjmcc.2009.12.020

5. Piroddi N, Belus A, Scellini B, Tesi C, Giunti G, Cerbai E, et al. Tension generation and relaxation in single myofibrils from human atrial and ventricular myocardium. Pflugers Arch. (2007) 454:63-73. doi: 10.1007/s00424-006-0181-3

6. Piroddi N, Belus A, Eiras S, Tesi C, van der Velden J, Poggesi C., et al. No direct effect of creatine phosphate on the cross-bridge cycle in cardiac myofibrils. Pflugers Arch. (2006) 452:3-6. doi: 10.1007/s00424-005-0008-7

7. Jeong MY, Walker JS, Brown RD, Moore RL, Vinson CS, Colucci WS., et al. AFos inhibits phenylephrine-mediated contractile dysfunction by altering phospholamban phosphorylation. Am J Physiol Heart Circ Physiol. (2010) 298:H1719-26. doi: 10.1152/ajpheart.00937.2009

8. Colomo F, Nencini S, Piroddi N, Poggesi C, Tesi C. Calcium dependence of the apparent rate of force generation in single striated muscle myofibrils activated by rapid solution changes. Adv Exp Med Biol. (1998) 453:373-81; discussion: 381-2. doi: 10.1007/978-1-4684-6039-1_42

9. Tesi C, Colomo F, Nencini S, Piroddi N, Poggesi C. The effect of inorganic phosphate on force generation in single myofibrils from rabbit skeletal muscle. Biophys J. (2000) 78:3081-92. doi: 10.1016/S0006-3495(00)76845-7

10. Tesi C, Colomo F, Nencini S, Piroddi N, Poggesi C. Modulation by substrate concentration of maximal shortening velocity and isometric force in single myofibrils from frog and rabbit fast skeletal muscle. J Physiol. (1999) 516 (Pt 3):847-53. doi: 10.1111/j.1469-7793.1999.0847u.x

11. Brenner B. Effect of $\mathrm{Ca} 2+$ on cross-bridge turnover kinetics in skinned single rabbit psoas fibers: implications for regulation of muscle contraction. Proc Natl Acad Sci USA. (1988) 85:3265-9. doi: 10.1073/pnas.85.9.3265

12. Jeong MY, Kinugawa K, Vinson C, Long CS. AFos dissociates cardiac myocyte hypertrophy and expression of the pathological gene program. Circulation (2005) 111:1645-51. doi: 10.1161/01.CIR.0000160367.99928.87

13. Satoh N, Suter TM, Liao R, Colucci WS. Chronic alpha-adrenergic receptor stimulation modulates the contractile phenotype of cardiac myocytes in vitro. Circulation (2000) 102:2249-54. doi: 10.1161/01.CIR.102.18.2249

14. Bugaisky LB, Zak R. Differentiation of adult rat cardiac myocytes in cell culture. Circ Res. (1989) 64:493-500. doi: 10.1161/01.RES.64.3.493

15. Volz A, Piper HM, Siegmund B, Schwartz P. Longevity of adult ventricular rat heart muscle cells in serum-free primary culture. J Mol Cell Cardiol. (1991) 23:161-73. doi: 10.1016/0022-2828(91)90103-S

16. Eppenberger ME, Hauser I, Baechi T, Schaub MC, Brunner UT, Dechesne $\mathrm{CA}$, et al. Immunocytochemical analysis of the regeneration of myofibrils

\section{ACKNOWLEDGMENTS}

The authors acknowledge Amit Patel for his assistance with writing the data acquisition program, Ted Anderson (Original Code Consultants) for his assistance in writing the data analysis program, and Allistair Moses for his assistance in designing the photodiode motion detector.

in long-term cultures of adult cardiomyocytes of the rat. Dev Biol. (1988) 130:1-15. doi: 10.1016/0012-1606(88)90408-3

17. Rust EM, Westfall MV, Metzger JM. Stability of the contractile assembly and $\mathrm{Ca} 2+$-activated tension in adenovirus infected adult cardiac myocytes. $\mathrm{Mol}$ Cell Biochem. (1998) 181:143-55. doi: 10.1023/A:1006802719136

18. Martin AF. Turnover of cardiac troponin subunits. Kinetic evidence for a precursor pool of troponin-I. J Biol Chem. (1981) 256:964-8.

19. Zak R, Martin AF, Prior G, Rabinowitz M. Comparison of turnover of several myofibrillar proteins and critical evaluation of double isotope method. J Biol Chem. (1977) 252:3430-5.

20. Demos-Davies KM, Ferguson BS, Cavasin MA, Mahaffey JH, Williams SM, Spiltoir JI, et al. HDAC6 contributes to pathological responses of heart and skeletal muscle to chronic angiotensin-II signaling. Am J Physiol Heart Circ Physiol. (2014) 307:H252-8. doi: 10.1152/ajpheart.00149.2014

21. O'Connor CM, Gattis WA, Uretsky BF, Adams KF, McNulty SE, Grossman $\mathrm{SH}$, et al. Continuous intravenous dobutamine is associated with an increased risk of death in patients with advanced heart failure: insights from the Flolan International Randomized Survival Trial (FIRST). Am Heart J. (1999) 138 (1 Pt 1):78-86. doi: 10.1016/S0002-8703(99)70250-4

22. Packer M, Carver JR, Rodeheffer RJ, Ivanhoe RJ, DiBianco R, Zeldis SM, et al. Effect of oral milrinone on mortality in severe chronic heart failure. The PROMISE study research group. N Engl J Med. (1991) 325:1468-75. doi: 10.1056/NEJM199111213252103

23. Endoh M, Shimizu T, Yanagisawa T. Characterization of adrenoceptors mediating positive inotropic responses in the ventricular myocardium of the dog. Br J Pharmacol. (1978) 64:53-61. doi: 10.1111/j.1476-5381.1978.tb08640.x

24. Walker JS, Walker LA, Margulies K, Buttrick P, de Tombe P. Protein kinase A changes calcium sensitivity but not crossbridge kinetics in human cardiac myofibrils. Am J Physiol Heart Circ Physiol. (2011) 301:H138-46. doi: 10.1152/ajpheart.00838.2010

25. Rao V, Cheng Y, Lindert S, Wang D, Oxenford L, McCulloch AD, et al. PKA phosphorylation of cardiac troponin I modulates activation and relaxation kinetics of ventricular myofibrils. Biophys J. (2014) 107:1196-204. doi: 10.1016/j.bpj.2014.07.027

26. Vikhorev PG, Song W, Wilkinson R, Copeland O, Messer AE, Ferenczi MA., et al. The dilated cardiomyopathy-causing mutation ACTC E361G in cardiac muscle myofibrils specifically abolishes modulation of $\mathrm{Ca}(2+)$ regulation by phosphorylation of troponin I. Biophys J. (2014) 107:2369-80. doi: $10.1016 /$ j.bpj.2014.10.024

Conflict of Interest Statement: The authors declare that the research was conducted in the absence of any commercial or financial relationships that could be construed as a potential conflict of interest.

Copyright (c) 2019 Woulfe, Ferrara, Pioner, Mahaffey, Coppini, Scellini, Ferrantini, Piroddi, Tesi, Poggesi and Jeong. This is an open-access article distributed under the terms of the Creative Commons Attribution License (CC BY). The use, distribution or reproduction in other forums is permitted, provided the original author $(s)$ and the copyright owner(s) are credited and that the original publication in this journal is cited, in accordance with accepted academic practice. No use, distribution or reproduction is permitted which does not comply with these terms. 